\title{
Simply longer is not better: reversal of theta burst after-effect with prolonged stimulation
}

\author{
Olga Lucía Gamboa • Andrea Antal • \\ Vera Moliadze • Walter Paulus
}

Received: 15 January 2010/Accepted: 4 May 2010/Published online: 22 June 2010

(c) The Author(s) 2010. This article is published with open access at Springerlink.com

\begin{abstract}
From all rTMS protocols at present, the theta burst stimulation (TBS) is considered the most efficient in terms of number of impulses and intensity required during a given stimulation. The aim of this study was to investigate the effects of inhibitory and excitatory TBS protocols on motor cortex excitability when the duration of stimulation was doubled. Fourteen healthy volunteers were tested under four conditions: intermittent theta bust stimulation (iTBS), continuous theta burst stimulation (cTBS), prolonged intermittent theta bust stimulation (ProiTBS) and prolonged continuous theta burst stimulation (ProcTBS). The prolonged paradigms were twice as long as the conventional TBS protocols. Conventional facilitatory iTBS converted into inhibitory when it was applied for twice as long, while the normally inhibitory cTBS became facilitatory when the stimulation duration was doubled. Our results show that TBS-induced plasticity cannot be deliberately enhanced simply by prolonging TBS protocols. Instead, when stimulating too long, after-effects will be reversed. This finding supplements findings at the short end of the stimulation duration range, where it was shown that conventional cTBS is excitatory in the first half and switches to inhibition only after the full length protocol. It is relevant for clinical applications for which an ongoing need for further protocol improvement is imminent.
\end{abstract}

Keywords rTMS - TBS - Motor cortex - Neuroplasticity · Brain stimulation · Human

O. L. Gamboa ( $\bigotimes)$ · A. Antal · V. Moliadze · W. Paulus Department of Clinical Neurophysiology, Georg-August University, Robert-Koch-Strasse 40, 37075 Göttingen, Germany e-mail: olgagamboa@med.uni-goettingen.de

\section{Introduction}

Non-invasive induction of neuroplastic processes by transcranial stimulation techniques is being increasingly implemented for the functional improvement of diseases and for enhancing or reducing cortical excitability and modifying related physiological activities in basic neuroscience research. Apart from transcranial direct current stimulation (tDCS), the most well-known method currently used to influence motor cortex (M1) excitability in the brain is the repetitive transcranial magnetic stimulation (rTMS) (Ziemann et al. 2008); extended to the more efficient variant termed theta burst stimulation (TBS) (Huang et al. 2005). TBS modulates M1 excitability in an inhibitory or excitatory way depending on the pattern of stimulation used. Intermittent TBS (iTBS) increases M1 excitability whereas continuous TBS (cTBS) decreases it as confirmed meanwhile on I-wave level (Di Lazzaro et al. 2005). So far after-effects of up to $1 \mathrm{~h}$ post-stimulation have been described (Gentner et al. 2008).

The ability to modulate M1 plasticity within 1-3 min makes TBS the most powerful tool for therapeutic applications in terms of application time. TBS has also shown its efficacy in other brain regions such as the visual (Franca et al. 2006), the somatosensory (Ishikawa et al. 2007) and premotor cortices (Mochizuki et al. 2005; Huang et al. 2009). Nevertheless, the variety of parameters such as number of pulses, stimulus intensity and time of stimulations needs further evaluation in order to optimize TBS for clinical applications.

The TBS paradigm consists of 3 pulses at $50-100 \mathrm{~Hz}$ repeated at $5 \mathrm{~Hz}$ (Huang et al. 2005). However, the total duration of the stimulation depends on the type of stimulation, since in iTBS successive interval times of $8 \mathrm{~s}$ add to a train of TB lasting $2 \mathrm{~s}$ summing up to $190 \mathrm{~s}$ in 
comparison with the cTBS total duration time of $40 \mathrm{~s}$. The interval of $8 \mathrm{~s}$ essentially determines the switch from inhibition to excitation with 600 stimuli.

Gentner et al. (2008) have shown that cTBS applied for $20 \mathrm{~s}$ (cTBS300 pulses) facilitated the amplitude of motor evoked potential (MEP) whereas when applied for $40 \mathrm{~s}$ (cTBS600 pulses), the amplitudes of MEPs were suppressed. However, when voluntary muscle contraction was performed before stimulation, both cTBS300 and cTBS600 induced inhibition. Furthermore, the duration of the aftereffects was 20 min when cTBS300 was applied and up to $1 \mathrm{~h}$ when cTBS600 was applied.

The aim of this study was to investigate the after-effects induced by longer TBS stimulation, in this case twice the classical paradigms: prolonged iTBS (ProiTBS) and prolonged cTBS (ProcTBS) on the corticospinal excitability. Compared to the previously applied protocols (Di Lazzaro et al. 2005, 2008; Huang et al. 2005; Gentner et al. 2008), we have doubled the duration of the stimulation without an additional interval and unexpectedly observed that the state of the cortical excitability was not only reduced hereby but could be changed to the opposite direction by modifying only this parameter.

\section{Methods}

Materials and methods

\section{Subjects}

Fourteen healthy subjects (seven women and seven men) between 21 and 27 years of age participated in this study. All subjects were informed about all aspects of the experiments, and all gave an informed consent.

All subjects were right-handed, according to the Edinburgh handedness inventory (Oldfield 1971) and homozygote with regard to BDNF polymorphism (Cheeran et al. 2008; Antal et al. 2010). For genotyping, blood samples were collected from each subject as previously described (Antal et al. 2010). Informed consent was obtained from each subject, before any experimental procedure was carried out. We conformed to the Declaration of Helsinki; and the experimental protocol was approved by the Ethics Committee of the University of Göttingen.

\section{MEP recordings}

In each subject, the experimental sessions were performed at the same time during the day. To assess excitability, motor evoked potentials (MEPs) of the right first dorsal interosseus muscle (FDI) were recorded following stimulation of its motor-cortical representation field by single-pulse TMS.
Magnetic stimulation was performed with a Magstim 200 magnetic stimulator (Magstim Company, Whiteland, Wales, UK). A figure-of-eight standard double magnetic coil (diameter of one winding, $70 \mathrm{~mm}$; peak magnetic field, $2.2 \mathrm{~T}$; average inductance, $16.35 \mu \mathrm{H}$ ) was held tangentially to the skull, with the handle pointing backwards and laterally at $45^{\circ}$ to the sagittal plane, resulting in a posterior-anterior direction of current flow in the brain.

Surface electromyogram (EMG) was recorded from the right $\mathrm{FDI}$ through a pair of $\mathrm{Ag}-\mathrm{AgCl}$ surface electrodes in a belly-tendon montage. Raw signals were amplified, bandpass filtered ( $2 \mathrm{~Hz}-2 \mathrm{kHz}$; sampling rate, $5 \mathrm{kHz}$ ), digitized with a micro 1401 AD converter (Cambridge Electronic Design, Cambridge, UK) controlled by Signal Software (Cambridge Electronic Design, version 2.13) and stored on a personal computer for off-line analysis. Complete relaxation was controlled through auditory and visual feedback of EMG activity and whenever it was necessary, the subject was instructed to relax.

Stimulus intensities (as percentage of maximal stimulator output) of TMS to evoke MEPs of $\sim 1 \mathrm{mV}$ peak-topeak amplitude were determined at the beginning of each experiment, and a baseline of TMS-evoked MEPs (25 stimuli) was recorded at $0.25 \mathrm{~Hz}$ before the stimulation. Active motor threshold (AMT) was defined as the lowest stimulus intensity at which five out of ten consecutive stimuli elicited reliable MEPs (above $200 \mu \mathrm{V}$ in amplitude) during isometric contraction of the contralateral FDI muscle (Rothwell et al. 1999). To determine AMT, the subjects were asked to press a ball $(8 \mathrm{~cm}$ diameter) connected to a display where the actual pressure values were quantified. We introduced an interval of $5 \mathrm{~min}$ before we started the experiments.

\section{TBS technique}

The TBS pattern was common for all protocols used in this study, and the stimulation was applied over the primary motor cortex.

TBS was delivered using a Magstim Super Rapid stimulator and TBS pattern consisted of bursts containing 3 pulses at $50 \mathrm{~Hz}$ repeated at $5 \mathrm{~Hz}$ and an intensity of $80 \%$ AMT. For iTBS, a $2 \mathrm{~s}$ train of TBS was repeated every $10 \mathrm{~s}$ for a total of $190 \mathrm{~s}$ (600 pulses) (Huang et al. 2005). In prolonged iTBS, the stimulation protocols consisted of 40 cycles that all together lasted $390 \mathrm{~s}$ with a total of 1200 pulses.

In the continuous theta burst stimulation paradigm, a $40 \mathrm{~s}$ train of uninterrupted TBS was given (600 pulses) (Huang et al. 2005), whereas in prolonged cTBS, a $80 \mathrm{~s}$ train of TBS was applied (1200 pulses), again without an interval between both sessions. 
Following stimulation, 25 single test-pulse MEPs were recorded at $0.25 \mathrm{~Hz}$, i.e. approximately $0 \mathrm{~min}$ and then every $10 \mathrm{~min}$ up to $60 \mathrm{~min}$ post-stimulation.

Four experimental sessions were performed, each of them with at least a free interval of 5 days to avoid carryover effects. The subjects received iTBS, ProiTBS, cTBS and ProcTBS in a randomized order.

\section{Statistics}

One way ANOVA was performed to check the magnitude and duration of after-effects of each TBS paradigm. Comparison among TBS paradigms was performed using repeated measures ANOVA (TYPE (iTBS vs. ProiTBS or cTBS vs. ProcTBS) $\times$ TIME of MEP recordings). Data were not normalized and were corrected for multiple testing using Bonferroni method. Sphericity was assumed according to Mauchly's test, and Greenhouse-Geisser correction was used when sphericity was violated. Statistical tests were carried out using SPSS version 17.0. (SPSS Inc., Chicago). $P$-values $\leq 0.05$ were considered to be statistically significant.

\section{Results}

No side effects were reported by the subjects after any of the experimental sessions.

\section{iTBS versus cTBS}

Repeated measures ANOVA verified classical behavior of both TBS paradigms as expected. Classical iTBS showed facilitation on M1 and classical cTBS displayed suppression. Differences were observed regarding the TYPE of stimulation $\left(F_{1,13}=42.957, P<0.001\right)$ and TYPE $\times$ TIME interaction $\left(F_{7}, 91=7.780, P<0.001\right)$. TIME of stimulation was not significant $\left(F_{7}, 9_{91}=0.838\right.$, $P=0.559$ ). Bonferroni test revealed significant differences between iTBS and cTBS at the time points $0 \mathrm{~min}$ until $50 \min (P<0.05)$ post-stimulation.

\section{ProiTBS versus ProcTBS}

Unexpectedly, prolonged paradigms showed a reversal in their after-effects compared to their correspondent classical protocols. ProiTBS and ProcTBS showed significant differences according to repeated measures ANOVA. These were observed in terms of TYPE of stimulation $\left(F_{1,13}=35.941 P<0.001\right)$ and TIME $\times$ TYPE interaction $\left(F_{7,91}=8.449 P \leq 0.001\right)$. The factor TIME did not show any significant effect $\left(F_{7,91}=1.661 P=0.128\right)$. The post hoc test showed statistically significant differences in

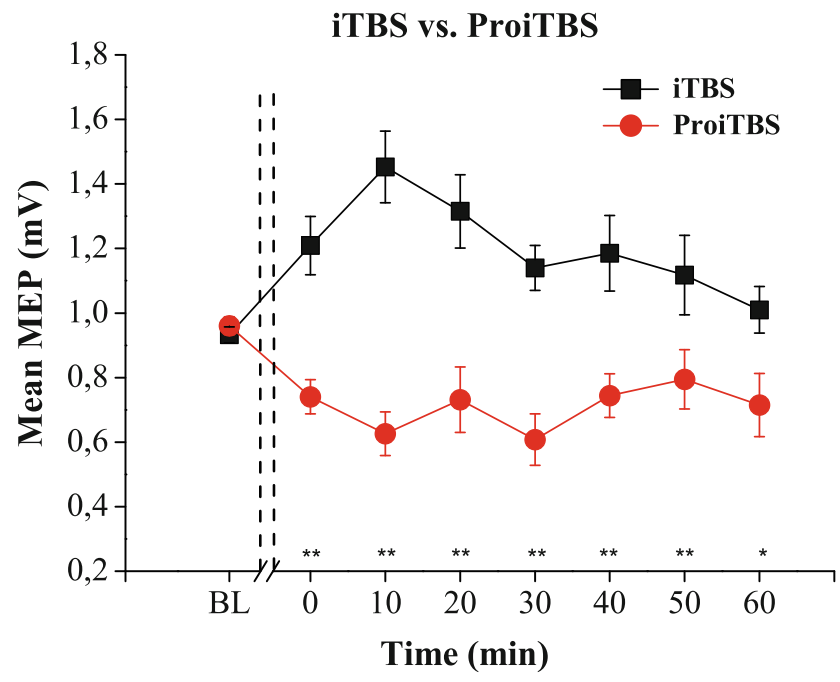

Fig. 1 Effects of intermittent theta burst stimulation (iTBS) and prolonged iTBS (ProiTBS) on the mean amplitude of motor evoked potentials (MEP) before and after stimulation. iTBS shows a classic excitatory behavior through an increase in the amplitude of the MEPs. In contrast, ProiTBS shows a reversed effect displaying inhibition in MEPs amplitude. Repeated measures ANOVA revealed that these differences are statistically significant $\left(F_{1}, 13=42.697, P<0.001\right)$. Error bars indicate standard error $(* * P<0.01)$

the time points from 0 min until 60 min after stimulation $(P \leq 0.05)$.

\section{iTBS versus ProiTBS}

iTBS showed a classical behavior and induced excitability increase (Fig. 1). One way ANOVA showed significant differences $\left(F_{7,91}=3.498, P=0.002\right)$. At the time point $10 \min (P=0.019)$, a significant difference was observed between baseline and post-stimulation MEP amplitudes according to Bonferroni test.

In contrast, ProiTBS suppressed MEPs amplitude $\left(F_{7,91}=3.646, P=0.002\right)$. Significant differences were seen in this paradigm when single points were compared to baseline at the time points $0 \mathrm{~min}(P=0.004), 10 \mathrm{~min}$ $(P=0.002), 30 \mathrm{~min}(P=0,010)$ and $40 \mathrm{~min}(P=0,028)$. (Data of mean MEP values of MEPs and motor thresholds are summarized in Table 1).

Repeated measures ANOVA revealed that excitatory after-effects on M1 due to iTBS are significantly different from the inhibitory after-effects induced by ProiTBS regarding the TYPE of stimulation $\left(F_{1},{ }_{13}=42.697\right.$, $P<0.001)$ and TYPE $\times$ TIME interaction $\left(F_{7,91}=6.230\right.$, $P=0.001)$. However, TIME of stimulation $\left(F_{7,91}=1.073\right.$, $P=0.387)$ was not statistically significant.

Bonferroni test revealed significant differences between iTBS and ProiTBS at the time points 0 min until $60 \mathrm{~min}$ $(P<0.05)$ post-stimulation (Fig. 1). 
Table 1 Motor thresholds and MEP amplitudes

\begin{tabular}{|c|c|c|c|c|c|c|c|c|c|}
\hline & \multirow[t]{3}{*}{ AMT [\% MSO] } & \multicolumn{8}{|c|}{ MEP Amplitude } \\
\hline & & \multirow[t]{2}{*}{ Baseline } & \multicolumn{7}{|c|}{ Time after stimulation (min) } \\
\hline & & & 0 & 10 & 20 & 30 & 40 & 50 & 60 \\
\hline cTBS & $47.57 \pm 2.01$ & $0.96 \pm 0.03$ & $0.79 \pm 0.06$ & $0.67 \pm 0.07$ & $0.61 \pm 0.04$ & $0.63 \pm 0.06$ & $0.76 \pm 0.07$ & $0.81 \pm 0.07$ & $0.88 \pm 0.11$ \\
\hline PcTBS & $49.14 \pm 2.13$ & $0.99 \pm 0.02$ & $1.45 \pm 0.13$ & $1.49 \pm 0.12$ & $1.46 \pm 0.09$ & $1.41 \pm 0.14$ & $1.35 \pm 0.09$ & $1.34 \pm 0.10$ & $1.06 \pm 0.10$ \\
\hline iTBS & $47.57 \pm 1.86$ & $0.93 \pm 0.03$ & $1.21 \pm 0.10$ & $1.45 \pm 0.11$ & $1.32 \pm 0.12$ & $1.14 \pm 0.07$ & $1.18 \pm 0.12$ & $1.12 \pm 0.13$ & $1.01 \pm 0.06$ \\
\hline PiTBS & $48.43 \pm 1.81$ & $0.96 \pm 0.03$ & $0.74 \pm 0.05$ & $0.63 \pm 0.07$ & $0.73 \pm 0.1$ & $0.60 \pm 0.08$ & $0.74 \pm 0.07$ & $0.79 \pm 0.09$ & $0.77 \pm 0.09$ \\
\hline
\end{tabular}

Motor thresholds and MEP amplitudes for baseline and post-stimulation measurements obtained for each TBS paradigm (mean \pm SEM). MSO, Maximum Stimulator Output

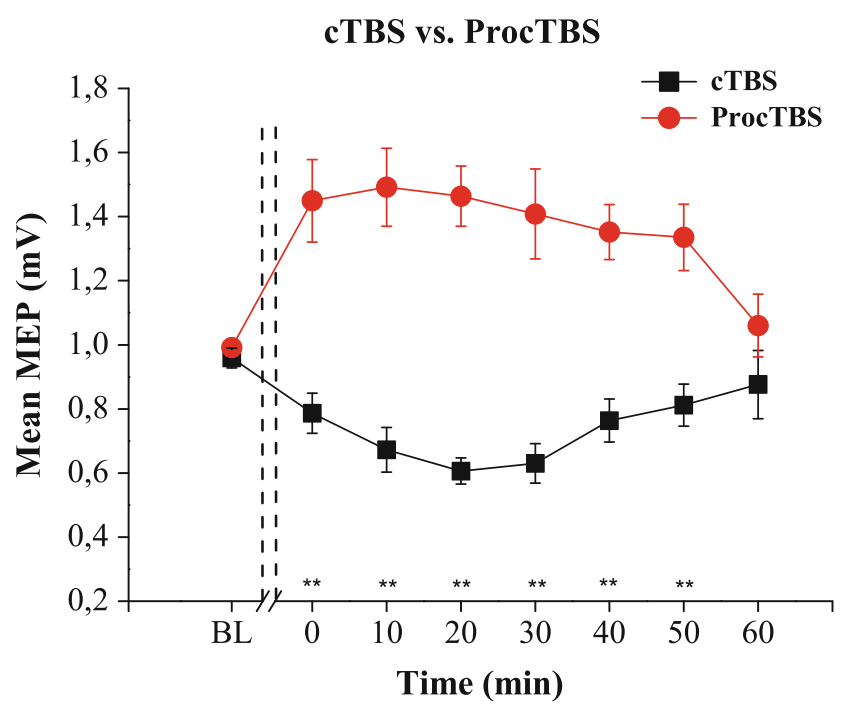

Fig. 2 Effects of continuous theta burst stimulation (cTBS) and prolonged cTBS (ProcTBS) on the mean amplitude of MEP. As expected, continuous TBS suppressed the amplitude of MEPs. ProcTBS, however, shows a reversed effect resulting in enhancement in MEPs amplitude. Repeated measures ANOVA indicates that the differences observed in these two techniques are statistically significant $\left(F_{1}, 13=36.762 P<0.001\right)$. Error bars indicate standard error (** $P<0.01)$

\section{cTBS versus ProcTBS}

As expected, suppression in the amplitude of MEPs was observed when cTBS was applied over M1 (See Table 1), $\left(F_{7,}, 91=4.730, P>0.001\right)$, (Fig. 2). Bonferroni test showed significant differences at the time points $10 \mathrm{~min}$ $(P=0,039), 20 \min (P<0.001)$ and $30 \min (P=0,001)$ compared to the baseline data.

In contrast, amplitudes of MEPs after ProcTBS were significantly increased $\left(F_{7,91}=5.761, P<0.001\right)$. Significant differences at the time points $10 \mathrm{~min}(P=0.027)$, $20 \mathrm{~min} \quad(P=0,006)$ and $40 \mathrm{~min} \quad(P=0,031)$ were observed when Bonferroni test was applied (See Fig. 2).

Significant differences between cTBS and ProcTBS on MEPS according to repeated measures ANOVA were observed in terms of TYPE of stimulation $\left(F_{1,13}=36.762\right.$ $P<0.001)$ and TIME $\times$ TYPE interaction $\left(F_{7,91}=9.065\right.$ $P<0.001)$. The factor TIME did not show any significant effect $\left(F_{7,91}=1.252 P=0.283\right)$.

The post hoc test showed statistically significant differences in the time points from 0 min until 50 min after stimulation $(P<0.001)$.

Data of mean MEP values of MEPs and motor thresholds for each paradigm are summarized in Table 1.

\section{Discussion}

The present study provides unexpected results in several ways. It was mainly designed as an explorative study to increase the effectiveness of TBS protocols for clinical studies, e.g. for treatment of depression and improving the motor disabilities in multiple sclerosis and Parkinson's disease. We expected the double duration protocols to be more effective with regard to the duration of the induced after-effects than the conventional protocol inaugurated by Huang et al. (2005).

First, surprisingly we found that less is more in terms of uninterrupted TBS duration, since doubling stimulation duration of TBS resulted in a reversal of effect with regard to cortical excitability. Second, we were astonished that stimulation effects not only ceased toward zero however they turned into the opposite direction. In a way, this might further corroborate the safety of iTBS with relation to induction of seizures (Paulus 2005). So far only one seizure has been reported with TBS, interestingly after 150 pulses of "inhibitory" cTBS, however, applied with increased intensity of $100 \%$ of resting motor threshold (RMT) at the motor cortex (Oberman and Pascual-Leone 2009). In the context of the present results, higher intensities require further studies to investigate if the reversal of inhibition to excitation with regard to cTBS occurs earlier if higher intensities are applied.

'Dose dependent' increase of the after-effects of rTMS is frequently reported in the literature. For instance, the 
amplitude of MEPs increased when 1800 stimuli at $5 \mathrm{~Hz}$ were applied but no significant after-effect was observed using only 150 stimuli (Peinemann et al. 2004). In another study, trains of $10 \mathrm{~Hz}$ rTMS applied for $1.5 \mathrm{~s}$ increased MEP amplitudes, whereas rTMS of the same frequency applied for 5 s decreased them (Jung et al. 2008). Hamada et al. (2008) introduced a new quadripulse paradigm (QPS). They have studied if the first part of a QPS series might have had a priming effect on the subsequent part of the rTMS. QPS at $5 \mathrm{~ms}$ ISI for $30 \mathrm{~min}$ increased cortical excitability but the same stimulation for $40 \mathrm{~min}$ had no effect, which may be explained by a priming effect of the first part of the stimulation leading to increased threshold for long-term potentiation (LTP) (Hamada et al. 2008).

In terms of TBS, a previous study has already reported that the excitatory or inhibitory nature of the after-effects depends on the number of pulses used during the stimulation (Gentner et al. 2008). cTBS applied for $20 \mathrm{~s}$ (300 pulses) is able to increase excitability in the corticospinal system but induces inhibition when the stimulation is applied for $40 \mathrm{~s}$ (600 pulses) (Gentner et al. 2008). However, to the best of our knowledge, there are no studies showing facilitatory effect due to prolonged inhibitory protocols and regarding iTBS, this is the first study, in which stimulation of this kind has been performed with a longer duration.

Concerning the possible underlying mechanisms of ProcTBS, Gentner et al. (2008) have suggested that reversal of the effects post-stimulation could be due to the combination of the number of TMS bursts and previous voluntary motor activation. The authors showed that cTBS applied for $20 \mathrm{~s}$ (300 pulses) increased corticospinal excitability but when isometric voluntary contraction was performed prior to stimulation, depression of MEPs amplitudes was observed. Indeed, we used AMT as a parameter to determine the magnetic stimulation intensities during TBS. AMT requires muscle contraction for 3-5 min in order to be established. We waited for $5 \mathrm{~min}$ before starting TBS in all 4 conditions in order to reduce possible after-effects of muscle innervation. Since iTBS and cTBS results were comparable with literature data and the ProiTBS and ProcTBS data were obtained under the same condition, we do not expect a relevant effect from the preinnervation data. We also paid attention to complete relaxation during TBS knowing that TBS exhibits different effects dependent on the size of voluntary muscle contraction (Ziemann et al. 2004; Gentner et al. 2008; Huang et al. 2008).

In a recent study, it was shown that also the excitatory after-effects of conventional $5 \mathrm{~Hz}$ stimulation require intervals (Rothkegel et al. 2010). If $5 \mathrm{~Hz}$ was applied continuously, after-effects switched into inhibition. Thus, in TBS as well as in rTMS research, a more general understanding of the importance of stimulation intervals in relation to stimulation duration is required. Also in rat hippocampal slice preparation, it was observed that TBS results in variable amounts of LTP depending on how many trains of stimulation were delivered (Abraham and Huggett 1997). In this study, an inverted U-shaped relation was observed between the applied trains of TBS and the degree of LTP. Over-stimulation inhibited for 60-90 min the subsequent induction of LTP by a normally efficient LTP-inducing protocol.

The mechanisms of repetitive TBS modulating corticospinal excitability are still unclear. Several different mechanisms may account for understanding our results, in first line homeostatic metaplasticity and gating/anti-gating processes. Homeostatic metaplasticity is an important physiological mechanism for the regulation of corticospinal excitability and synaptic plasticity (Brighina et al. 2010; Hamada et al. 2009). Indications that the previous history of neural activity of the stimulated zone is a critical factor which will determine the direction of the response obtained due to the type of stimulation has been provided by different studies (Hamada et al. 2008, 2009).According to the Bienenstock-Cooper-Munro (BCM) theory (Bienenstock et al. 1982), LTP might be favored by prolonged low levels of postsynaptic activity decrease. In the same way, LTD will be increased when there has been a previous history of enhanced postsynaptic activity. One possibility for explaining our results may be that prolonged TBS paradigms behave in a similar way as preconditioning-conditioning protocols. Thus, the first half (corresponding to the classical TBS) would serve as a preconditioning stimulus or priming and the second half as conditioning stimulus. The integration of the results due to both interventions would induce homeostatic BCM-like mechanisms (Potter-Nerger et al. 2009). The stimulation-induced after-effects would be the result of competition processes due to the incoming patterns and the states (stable or labile) of cortical neurones in the stimulated zone (Changeux et al. 1973; Bienenstock et al. 1982; Hamada et al. 2008).

However, "gating" processes (Ziemann and Siebner 2008; Siebner 2010) could be also considered as a means of interpreting the outcomes observed in this study. It is assumed that cTBS and iTBS involve LTD/LTP processes at cortical synapses (Di Lazzaro et al. 2005). These phenomena are controlled by $\mathrm{Ca} 2 \pm$ levels in the postsynaptic cell (Huang et al. 2008) and might be caused by a decrease in the strength of intracortical inhibition due to gating mechanisms (Ziemann and Siebner 2008; Siebner 2010).

When prolonged cTBS is applied in the first train of stimulation (priming stimulus), cTBS-induced LTD-like phenomena arising during this time may lead to an initial decrease in excitability. When gating occurs the levels of $\mathrm{Ca} 2+$ inside the targeted neurons are increased during the 
conditioning protocol inducing corticospinal excitability. In contrast, when stimulating the M1 with ProiTBS, the classical iTBS intervention will produce LTP-like effects promoting in the first instance facilitation, a "gating intervention" might reverse the sign of plasticity from LTP-like to LTD-like plasticity during the condition protocol, due to a decrease in the amount of calcium influx, favoring LTD-like plasticity (Siebner 2010). This explanation is supported by the fact that conventional TBS protocols have been shown to produce decreased or increased SICI (Huang et al. 2005). Indeed, if cTBS produces decreased SICI, subsequent rTMS protocol might be more effective in exciting cortical output neurons transsynaptically. By contrast, if iTBS produces increased SICI and leading to subsequent TBS less effective in exciting neurons, this result in a small amount of calcium influx by TBS protocol, favoring LTD-like plasticity.

In summary, our results show the possibility of inducing changes in M1 excitability to opposite directions applying the same stimulation paradigm but varying the duration of the stimulation (number of pulses) here, for longer stimulation durations. These results are relevant for optimizing clinical stimulation protocols.

Acknowledgments Supported by the German Ministry of Health (BMBF 01GQ0810 (OG), 01GQ 0782 (VM)) and the Rose Foundation (T298/14375/2004 and T298/14376/2004) and the Ministry of Education and Culture for Lower Saxony (ZN 2187).

Conflict of interest statement The authors have no financial or personal conflicts of interest.

Open Access This article is distributed under the terms of the Creative Commons Attribution Noncommercial License which permits any noncommercial use, distribution, and reproduction in any medium, provided the original author(s) and source are credited.

\section{References}

Abraham WC, Huggett A (1997) Induction and reversal of long-term potentiation by repeated high-frequency stimulation in rat hippocampal slices. Hippocampus 7:137-145

Antal A, Chaieb L, Moliadze V, Monte-Silva K, Poreisz C, Thirugnanasambandam N, Nitsche MA, Shoukier M, Ludwig H, Paulus W (2010) Brain-derived neurotrophic factor (BDNF) gene polymorphisms shape cortical plasticity in humans. Brain Stimulat (in press)

Bienenstock EL, Cooper LN, Munro PW (1982) Theory for the development of neuron selectivity: orientation specificity and binocular interaction in visual cortex. J Neurosci 2:32-48

Brighina F, Palermo A, Daniele O, Aloisio A, Fierro B (2010) Highfrequency transcranial magnetic stimulation on motor cortex of patients affected by migraine with aura: a way to restore normal cortical excitability? Cephalalgia 30:46-52

Changeux JP, Courrege P, Danchin A (1973) A theory of the epigenesis of neuronal networks by selective stabilization of synapses. Proc Natl Acad Sci USA 70:2974-2978
Cheeran B, Talelli P, Mori F, Koch G, Suppa A, Edwards M, Houlden H, Bhatia K, Greenwood R, Rothwell JC (2008) A common polymorphism in the brain-derived neurotrophic factor gene (BDNF) modulates human cortical plasticity and the response to rTMS. J Physiol 586:5717-5725

Di Lazzaro V, Pilato F, Saturno E, Oliviero A, Dileone M, Mazzone P, Insola A, Tonali PA, Ranieri F, Huang YZ, Rothwell JC (2005) Theta-burst repetitive transcranial magnetic stimulation suppresses specific excitatory circuits in the human motor cortex. J Physiol 565:945-950

Di Lazzaro V, Pilato F, Dileone M, Profice P, Oliviero A, Mazzone P, Insola A, Ranieri F, Meglio M, Tonali PA, Rothwell JC (2008) The physiological basis of the effects of intermittent theta burst stimulation of the human motor cortex. J Physiol 586:3871-3879

Franca M, Koch G, Mochizuki H, Huang YZ, Rothwell JC (2006) Effects of theta burst stimulation protocols on phosphene threshold. Clin Neurophysiol 117:1808-1813

Gentner R, Wankerl K, Reinsberger C, Zeller D, Classen J (2008) Depression of human corticospinal excitability induced by magnetic theta-burst stimulation: evidence of rapid polarityreversing metaplasticity. Cereb Cortex 18:2046-2053

Hamada M, Terao Y, Hanajima R, Shirota Y, Nakatani-Enomoto S, Furubayashi T, Matsumoto H, Ugawa Y (2008) Bidirectional long-term motor cortical plasticity and metaplasticity induced by quadripulse transcranial magnetic stimulation. J Physiol 586:3927-3947

Hamada M, Hanajima R, Terao Y, Okabe S, Nakatani-Enomoto S, Furubayashi T, Matsumoto H, Shirota Y, Ohminami S, Ugawa Y (2009) Primary motor cortical metaplasticity induced by priming over the supplementary motor area. J Physiol 587:4845-4862

Huang YZ, Edwards MJ, Rounis E, Bhatia KP, Rothwell JC (2005) Theta burst stimulation of the human motor cortex. Neuron 45:201-206

Huang YZ, Rothwell JC, Edwards MJ, Chen RS (2008) Effect of physiological activity on an NMDA-dependent form of cortical plasticity in human. Cereb Cortex 18:563-570

Huang YZ, Rothwell JC, Lu CS, Wang J, Weng YH, Lai SC, Chuang WL, Hung J, Chen RS (2009) The effect of continuous theta burst stimulation over premotor cortex on circuits in primary motor cortex and spinal cord. Clin Neurophysiol 120:796-801

Ishikawa S, Matsunaga K, Nakanishi R, Kawahira K, Murayama N, Tsuji S, Huang YZ, Rothwell JC (2007) Effect of theta burst stimulation over the human sensorimotor cortex on motor and somatosensory evoked potentials. Clin Neurophysiol 118:10331043

Jung SH, Shin JE, Jeong YS, Shin HI (2008) Changes in motor cortical excitability induced by high-frequency repetitive transcranial magnetic stimulation of different stimulation durations. Clin Neurophysiol 119:71-79

Mochizuki H, Franca M, Huang YZ, Rothwell JC (2005) The role of dorsal premotor area in reaction task: comparing the "virtual lesion" effect of paired pulse or theta burst transcranial magnetic stimulation. Exp Brain Res 167:414-421

Oberman LM, Pascual-Leone A (2009) Letter to the editor: report of seizure induced by continuous theta burst stimulation. Brain Stimulat 2:246-247

Oldfield R (1971) The assessment and analysis of handedness: the Edinburgh inventory. Neuropsychologia 9:97-113

Paulus W (2005) Toward establishing a therapeutic window for rTMS by theta burst stimulation. Neuron 45:181-183

Peinemann A, Reimer B, Loer C, Quartarone A, Munchau A, Conrad B, Siebner HR (2004) Long-lasting increase in corticospinal excitability after 1800 pulses of subthreshold $5 \mathrm{~Hz}$ repetitive TMS to the primary motor cortex. Clin Neurophysiol 115:1519_ 1526 
Potter-Nerger M, Fischer S, Mastroeni C, Groppa S, Deuschl G, Volkmann J, Quartarone A, Munchau A, Siebner HR (2009) Inducing homeostatic-like plasticity in human motor cortex through converging corticocortical inputs. J Neurophysiol 102:3180-3190

Rothkegel H, Sommer M, Paulus W (2010) Breaks during 5 Hz rTMS are essential for facilitatory after effects. Clin Neurophysiol 121:426-430

Rothwell JC, Hallett M, Berardelli A, Eisen A, Rossini P, Paulus W (1999) Magnetic stimulation: motor evoked potentials. The International Federation of Clinical Neurophysiology. Electroencephalogr Clin Neurophysiol Suppl 52:97-103
Siebner HR (2010) A primer on priming the human motor cortex. Clin Neurophysiol 121:461-463

Ziemann U, Siebner HR (2008) Modifying motor learning through gating and homeostatic metaplasticity. Brain Stimulat 1:60-66

Ziemann U, Ilic TV, Pauli C, Meintzschel F, Ruge D (2004) Learning modifies subsequent induction of long-term potentiation-like and long-term depression-like plasticity in human motor cortex. J Neurosci 24:1666-1672

Ziemann U, Paulus W, Nitsche MA, Pascual-Leone A, Byblow WD, Berardelli A, Siebner HR, Classen J, Cohen LG, Rothwell JC (2008) Consensus: motor cortex plasticity protocols. Brain Stimulat 1:164-182 